\title{
The Impact of Pain Invisibility on Patient-Centered Care and Empathetic Attitude in Chronic Pain Management
}

\author{
Emilie Paul-Savoie $\mathbb{D}^{1},{ }^{1}$ Patricia Bourgault, ${ }^{1}$ Stéphane Potvin $\mathbb{D}^{1},{ }^{2}$ Emilie Gosselin, ${ }^{1}$ \\ and Sylvie Lafrenaye $\mathbb{1}^{3}$ \\ ${ }^{1}$ School of Nursing, Faculty of Medicine and Health Sciences, Université de Sherbrooke, Sherbrooke, QC, Canada \\ ${ }^{2}$ Centre de Recherche de L'Institut Universitaire en Santé Mentale de Montréal, Department of Psychiatry, Faculty of Medicine, \\ Université de Montréal, Montreal, QC, Canada \\ ${ }^{3}$ Department of Pediatrics, Faculty of Medicine and Health Sciences, Université de Sherbrooke, Sherbrooke, QC, Canada
}

Correspondence should be addressed to Sylvie Lafrenaye; sylvie.lafrenaye@usherbrooke.ca

Received 11 April 2018; Accepted 29 August 2018; Published 24 September 2018

Academic Editor: Carlo Lai

Copyright $\odot 2018$ Emilie Paul-Savoie et al. This is an open access article distributed under the Creative Commons Attribution License, which permits unrestricted use, distribution, and reproduction in any medium, provided the original work is properly cited.

Objectives. The use of interdisciplinary patient-centered care (PCC) and empathetic behaviour seems to be a promising avenue to address chronic pain management, but their use in this context seems to be suboptimal. Several patient factors can influence the use of PCC and empathy, but little is known about the impact of pain visibility on these behaviours. The objective of this study was to investigate the influence of visible physical signs on caregiver's patient-centered and empathetic behaviours in chronic pain context. Methods. A convenience sample of 21 nurses and 21 physicians participated in a descriptive study. PCC and empathy were evaluated from self-assessment and observer's assessment using a video of real patients with chronic pain. Results. The results show that caregivers have demonstrated an intraindividual variability: PCC and empathetic behaviours of the participants were significantly higher for patients who have visible signs of pain (rheumatoid arthritis and complex regional pain syndrome) than for those who have no visible signs (Ehler-Danlos syndrome and fibromyalgia) $(p<0.001)$. Participants who show a greater difference in their patient-centered behaviour according to pain visibility have less clinical experience. Discussion. The pain visibility in chronic pain patients is an important factor contributing to an increased use of PCC and empathy by nurses and physicians, and clinical experience can influence their behaviours. Thus, pain invisibility can be a barrier to quality of care, and these findings reinforce the relevance to educating caregivers to these unconscious biases on their behaviour toward chronic pain patients.

\section{Introduction}

Chronic pain is a common public global health problem, associated with significant disability and many social consequences [1]. Chronic pain affects people of all ages, with a particularly high prevalence in adults, ranging from 10 to $55 \%$ [2]. People with chronic pain are known to consult health professional frequently, leading in a heavy economic burden $[3,4]$. To be effective, the treatment of chronic pain must consider biological, psychological, and social factors simultaneously [5]. Thus, the use of interdisciplinary patient-centered care (PCC) seems to be a promising avenue to address chronic pain management $[6,7]$.

There are many definitions of PCC in the context of nursing and medicine, but four dimensions are common to most cited definitions: patient-as-person, biopsychosocial perspective, sharing power and responsibility, and therapeutic alliance [8-10]. The use of this approach is related with many clinical benefits for patients with chronic pain [7, 11-13]. More specifically, researchers have shown that PCC resulted in a decrease in the number of tender points and psychological distress in fibromyalgia patients [11]. Qualitative findings support that PCC allows nurses to improve their assessment and to provide better anticipatory guidance and coaching [12].

In addition, many researchers suggest that empathy is necessary for the use of PCC $[8,14,15]$. In the model of Bilodeau et al., "having an empathetic presence" is an important dimension of PCC [8]. In healthcare, empathy is defined as a cognitive attribute involving an understanding 
of the patient's experience and perspective, as a separate individual, combined with an ability to communicate that understanding to the patient [16]. Interestingly, it has been suggested that high levels of empathy were related to positive outcomes for patients [17-19] and especially with chronic pain patients [20].

Although many studies support the benefits of PCC, [21] its use in chronic pain management seems to be challenging and suboptimal [20, 22]. Patient-centered behaviour or communication does not necessarily translate into a "unique recipe," and caregivers seem to use a flexible style according to patient characteristics [23]. Over the last years, it has been suggested that several patient factors can influence the use of PCC, such as age, gender, and level of education [24-26]. For example, caregivers would tend to use more PCC behaviour with women, older, and nonsmoker patients $[24,26]$. Moreover, some authors have shown that physicians tend to use more PCC when patients reported more physical symptoms, such as nausea, dry mouth, or constipation, and when they rated patients' health condition as more severe [26]. However, in chronic pain context, little is known about the impact of the "visibility" of physical signs on patient-centered behaviour.

Patient factors may also influence caregivers' empathy. Indeed, both behavioral and functional neuroimaging measures have demonstrated that some stigma or prejudice could modulate empathy [27]. Since many patients with chronic pain do not display any visible physical symptoms and remain stoic when they feel pain [28], it is important to assess the impact of this specific factor on patient-centered care and empathetic behaviour.

Various methods have been used for measuring the use of PCC and empathy, such as self-rating and observer rating $[9,23,29]$. Self-assessment instruments are the most common strategy used for measuring PCC and empathy, but they do not consider the influence of different patient factors such as the presence or absence of visible physical signs. The observation of PCC and empathy in real clinical encounters may raise some ethical and methodological issues, including the inability to have standardized visits, which introduces confounding variables. The use of standardized patient simulations is a very expensive strategy [30]. Videos of real patients with chronic pain could overcome these limitations by allowing a standardized and repetitive assessment of attitudes and behaviours of caregivers [31].

Thus, the main objective of this study was to investigate the influence of the presence of visible physical signs on patient-centered and empathetic behaviours of nurses and physicians using videos of real patients suffering from chronic pain. In view of the limited knowledge on the topic, we also sought to identify, in an exploratory fashion, the characteristics of the caregivers who respond differently to the presence or absence of visible signs of pain.

\section{Materials and Methods}

2.1. Study Design and Settings. This study is part of a larger study which investigated PCC and empathy in chronic pain management [32]. A descriptive design was used, conducted from May to November 2013, in the province of Quebec, Canada. The Scientific and Human Ethics Committee of the institution where the study took place approved the research protocol. A population composed of nurses and physicians has been targeted since interdisciplinary pain management is recommended [8]. A convenience sampling approach was chosen, and participants were recruited through advertisements and referrals. Participants were recruited from 16 different healthcare centres, including urban, semiurban, and rural centres, in the province of Quebec, Canada.

2.2. Participants. All participants gave their written, informed consent, and a coding system was used to keep the data confidential. To take part in the study, the participants need to (i) be active members of the Quebec Board of Nurses or the Quebec College of Physicians, (ii) have chronic pain patients in their routine practice, and (iii) speak French. After signing the informed consent, a sample of 21 nurses and 21 physicians took part in the study. A minimum of 38 participants was needed to detect a moderate difference $(d=0.5)$ between visible and invisible pain conditions with a power of $85 \%$ and a type- 1 error of $5 \%$. Participants did not know the detailed purpose of the study to avoid social desirability bias, but they were informed that pain management was investigated.

2.3. Self-Assessment Measures. The practice orientation of each participant was measured with the French version of the Patient Practitioner Orientation Scale (F-PPOS) [33]. This self-administrated questionnaire is designed for the assessment of practitioners' or future-practitioners' attitudes and orientations in their care approach. This scale contains 18 items divided into two subscales: "sharing" and "caring," and the four dimensions of PCC are represented. For each item, the caregiver is asked to indicate his or her level of agreement on a 6-point Likert scale (strongly agree to strongly disagree). A total score, ranging from patientcentered (a score of 6.00) to disease-centered (a score of $1.00)$, can be calculated with the addition of the two subscales. The original version of the PPOS has good face validity and acceptable internal consistency for the total scale (an alpha of 0.89) [34]. The French version of the PPOS has good content validity and acceptable internal consistency for the total scale an (alpha of 0.60) [34], since the minimum threshold is 0.50 [35].

The self-rated empathy was measured with the French version of the Jefferson Scale of Physician Empathy (F-JSPE) (P. Bourgault, S. Lavoie, and M. Grégoire et al., unpublished data, June 2009). This self-administrated questionnaire comprises 20 items divided into 4 dimensions: (i) adopting the patient's perspective, (ii) understanding the patient's experiences, feelings, and signals, (iii) ignoring the patient's perspective, and (iv) adopting the patient's way of thinking. The F-JSPE also includes a single item measuring the value placed on empathy. Participants respond to items on a 7 Likert scale. The total score, ranging from not empathic (a score of 20) to empathic (score of 140), can be calculated with the addition of all the items. The original version of the 
JSPE has good criterion-related validity with the Empathic Concern Scale of the Interpersonal Reactivity Index $(r=0.40)$, internal consistency (an alpha of 0.87 to 0.89 ), test-retest reliability (test-retest reliability coefficient $=0.65$ ), and construct validity $[16,36]$. The F-JSPE has good content validity and acceptable internal consistency (an alpha of 0.77) (P. Bourgault, S. Lavoie, and M. Grégoire et al., unpublished data, June 2009).

2.4. Observers' Assessment Measures. The PCC behaviour of each participant was measured with the Sherbrooke Observation Scale of patient-centered care (SOS-PCC) [31]. This instrument has been developed for the assessment of PCC behaviour of a professional caregiver by a trained observer, in the experimental clinical setting. Nine items divided into 4 dimensions (patient-as-person, biopsychosocial perspective, sharing power and responsibility, and therapeutic alliance) are measured on a 4-points Likert scale. The instrument was originally developed in French for a population of nurses and physicians. A total score, ranging from disease-centered (a score of 9) to patient-centered (a score of 36), can be calculated by the addition of all items. This instrument has good content validity, internal consistency (an alpha of 0.88 ), and inter-rater reliability (an intraclass coefficient (ICC) of 0.93) [31].

An adapted French version of Reynolds Empathy Scale (F-RES) has been used for the assessment of empathetic behaviour by a trained observer [37]. The F-RES consists of 9 items with a categorical rating scale ("yes," "no," or "incomplete"). This instrument has good internal consistency (an alpha of 0.70) and inter-rater reliability (an ICC of 0.85) [38]. In this study, we used a 7-item version with a 4-point Likert scale to make it more responsive to the context of videos. A total score, ranging from not empathetic (a score of 7) to empathetic (a score of 28), can be calculated by adding all items.

2.5. Procedure. All study participants watched four videos of real patients with chronic pain and were subsequently interviewed individually. These 4-minute videos showed female patients aged between 16 and 45 years-old, with different pathologies in which chronic pain was experienced (rheumatoid arthritis, complex regional pain syndrome (CRPS), Ehlers-Danlos syndrome, and fibromyalgia). A more detailed description of the development and content of these videos is available elsewhere [31]. In these videos, some patients had no visible physical signs (Ehlers-Danlos syndrome and fibromyalgia) and others had obvious visible deformities in the upper limb (rheumatoid arthritis and CRPS). After viewing the videos, participants had to describe the management plan that they would provide for each patient, and these answers were video recorded. At the end of the session, each participant responded to the self-administrated questionnaires. After the data collection ( $n=42$ participants), three external observers watched the recorded interviews of each participant (4 interviews/participant). The group of observers consisted of a resident in psychiatry, a nurse, and a $\mathrm{PhD}$ student in the healthcare field. The observers were selected based on their experience in the healthcare field (more than five years) and their complementary expertise (medicine, nursing, and research). To ensure the standardization in their assessment, they had previously been trained by the research team to complete the observation scale. They evaluated the PCC and empathetic behaviour demonstrated by participants for each video using the SOS-PCC and the F-RES. Figure 1 showed the conduct of the study.

2.6. Data Analysis. Statistical analyzes were performed using the software SPSS version 18.0. To describe continuous variables, mean (standard deviation) was used, whereas frequency (percentage) was used for nominal and categorical variables. To compare patient-centered and empathetic behaviour of the participants $(n=42)$ according to the presence or absence of visible physical signs in the patients presented in the videos, paired $T$-tests were used on the mean score of the three observers.

To investigate if the clinical experience can influence the modification of the behaviours according to the pain visibility, for each study participant, we calculated the difference of SOS-PCC and F-RES between the his/her behaviour for the patients with visible signs and the patients without visible signs. We divided the participants into two groups according to these differences: the participants who rated the two groups of patients similarly and those who rated the two groups of patients differently. To determine whether a participant showed similar or different behaviours, we have used the mean scores of SOS-PCC and F-RES for the thresholds. We used paired T-tests to compare the differences in clinical experience between the two groups study participants. The statistical level of significance was set at $p<0.05$. We do not have any missing data.

\section{Results and Discussion}

3.1. Participants' Characteristics. The sample included 42 native French-speaking caregivers ranging from 27 to 67 years $(M=46.12$ years; $\mathrm{SD}=10.84)$ and the majority was women (69\%). Twenty-one (50\%) of the participants were nurses, and $21(50 \%)$ were physicians. Our sample was composed of nurses working in different settings, general practitioners, and medical specialists (physiatrist, rheumatologist, orthopaedist, radiologist, nephrologist, neurologist, and psychiatrist). The nurses and physicians had an average of 19.74 years of clinical experience. In this group, participants self-reported a mean overall orientation for PPC of 4.82. More specifically, the mean was 4.49 for the sharing subscale and 5.16 for the caring subscale. The participants self-reported a mean of 116.53 for empathy. These results suggest moderate levels of PCC and empathy when participants assess themselves. For the observer's assessment with observation scales, the results are presented for each video. For observed PCC, the mean for the 4 videos was 25.94, and for observed empathy, the mean was 20.70 . Table 1 shows the characteristics of these participants. 


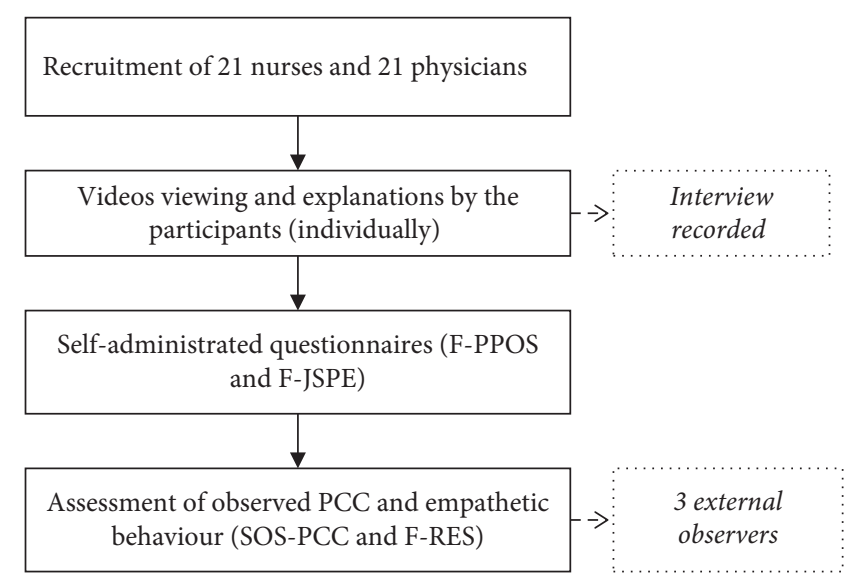

FIgURE 1: Conduct of the study. F-PPOS indicates the French version of the Patient-Practitioner Orientation Scale, F-JSPE indicates the French version of the Jefferson Scale of Physician Empathy, PCC indicates patient-centered care, SOS-PCC indicates Sherbrooke Observation Scale of patient-centered care, and F-RES indicates the French version of the Reynolds Empathy Scale.

\subsection{Patient-Centered and Empathetic Behaviour according to} the Presence or Absence of Visible Physical Signs. In total, 168 interviews in response to the videos were videotaped successfully. We divided these interviews in two groups: (i) interviews in response to the videos presenting patients with visible physical signs (rheumatoid arthritis and CRPS) and (ii) interviews in response to the videos presenting patients without visible physical signs (Ehlers-Danlos syndrome and fibromyalgia). Regarding the observed patient-centered and empathetic behaviours, the mean for SOS-PCC and F-RES was calculated for both groups. The results support that patient-centered and empathetic behaviour was significantly higher for the group of patients with visible physical signs $(p<0.001)$ (Figure 2). The group of participants who show a greater difference in their patient-centered behaviour according to pain visibility have less clinical experience than the group that had similar behaviour for both patients with and without physical visible signs $(p=0.03)$. For empathetic behaviour, the difference is not statistically significant $(p=0.23)$ (Table 2$)$.

\section{Discussion}

The results suggest that our sample of caregivers have moderate levels of self-reported PCC and empathy. In comparison with other studies, nurses and physicians who have participated in our study used more PCC than other groups of nurses and physicians with similar sociodemographic characteristics $[38,39]$. For self-reported empathy, our sample was comparable to other nurses and physicians [40].

The main purpose of this descriptive study was to investigate the influence of the presence or absence of visible physical signs on caregiver behaviours and attitudes in chronic pain management. PCC and empathy were assessed from two perspectives, using a combination of self-administrated questionnaires and observation scales using innovative videos of real patients with chronic pain. Our results show that nurses and physicians show intraindividual variability. Indeed, we found that PCC and empathetic behaviour of nurses and physicians vary according to the presence or absence of visible physical signs in patients with chronic pain.

Interestingly, another research team have demonstrated that residents in internal medicine have displayed more patient-centered behaviour when they consider that the patient has a more severe health condition and more visible physical symptoms [26]. In the same vein, another research group has demonstrated that patient-centered practice style of physicians was positively related with higher patient selfreported physical health status [24]. Although the severity of the disease is not automatically associated with the presence of visible physical symptoms, the results of clinical observations have shown that patients with more severe diseases were given longer consultations and the opportunity to talk more about their medical condition [41]. These observations have a great importance in the context of chronic pain, since these patients often have no visible physical signs, and can remain stoic when they feel pain [28] because their condition is often present for several years. For example, patients with neuropathic pain suffer from allodynia and hyperalgesia, but they often have no obvious physical signs. Moreover, some authors have suggested that professional caregivers were baffled by the lack of correlation between pain intensity verbally and nonverbally expressed by chronic pain patients and their medical condition [42].

It has been shown that physicians tend to rely on their initial assessment of the pain of their patients with chronic pain even when this initial assessment underestimates the pain subsequently reported verbally by the patients [43]. Thus, if chronic pain patients are not asked about their preoccupations and their medical condition's severity, stoic patients and those with less visible physical signs are more likely to receive suboptimal personalized care, with a more disease-centered orientation and less empathetic behaviour. These findings suggest that nurses and physicians adjust their practice orientation and empathy according to the clinical condition. It could explain why many patients with chronic pain are frustrated after their clinical encounters. This has a particular impact since these interactions are prominent in their experience [43]. 
TABLE 1: Results of self-administrated questionnaires.

\begin{tabular}{lr}
\hline & Participants $(n=42)$ \\
\hline Age, mean (SD) & $46.12(10.84)$ \\
Gender, $n$ (\%) & $13(31)$ \\
Male & $29(69)$ \\
Female & $21(50)$ \\
Profession, $n$ (\%) & $21(50)$ \\
Nurse & $19.74(10.34)$ \\
Physician & $4.82(0.39)$ \\
Clinical experience, mean (SD) & $4.49(0.59)$ \\
F-PPOS total, mean (SD) & $5.16(0.37)$ \\
Sharing, mean (SD) & $116.53(9.80)$ \\
Caring, mean (SD) & $25.94(3.86)$ \\
F-JSPE, mean (SD) & $27.12(3.88)$ \\
SOS-PCC, mean (SD) & $27.38(4.30)$ \\
Patient with rheumatoid arthritis, mean (SD) & $26.02(4.38)$ \\
Patient with CRPS, mean (SD) & $24.87(5.11)$ \\
Patient with Ehlers-Danlos syndrome, mean (SD) & $20.70(3.41)$ \\
Patient with fibromyalgia, mean (SD) & $21.89(3.59)$ \\
F-RES, mean (SD) & $22.30(3.25)$ \\
Patient with rheumatoid arthritis, mean (SD) & $20.34(3.81)$ \\
Patient with CRPS, mean (SD) & $18.94(4.05)$ \\
Patient with Ehlers-Danlos syndrome, mean (SD) & \\
Patient with fibromyalgia, mean (SD) & \\
\hline
\end{tabular}

$\mathrm{SD}=$ standard deviation; F-PPOS = French version of Patient-Practitioner Orientation Scale; F-JSPE = French version of Jefferson Scale of Physician Empathy; SOS-PCC = Sherbrooke Orientation Scale of patient-centered care; CRPS = complex regional pain syndrome; F-RES = French version of Reynolds Empathy Scale.

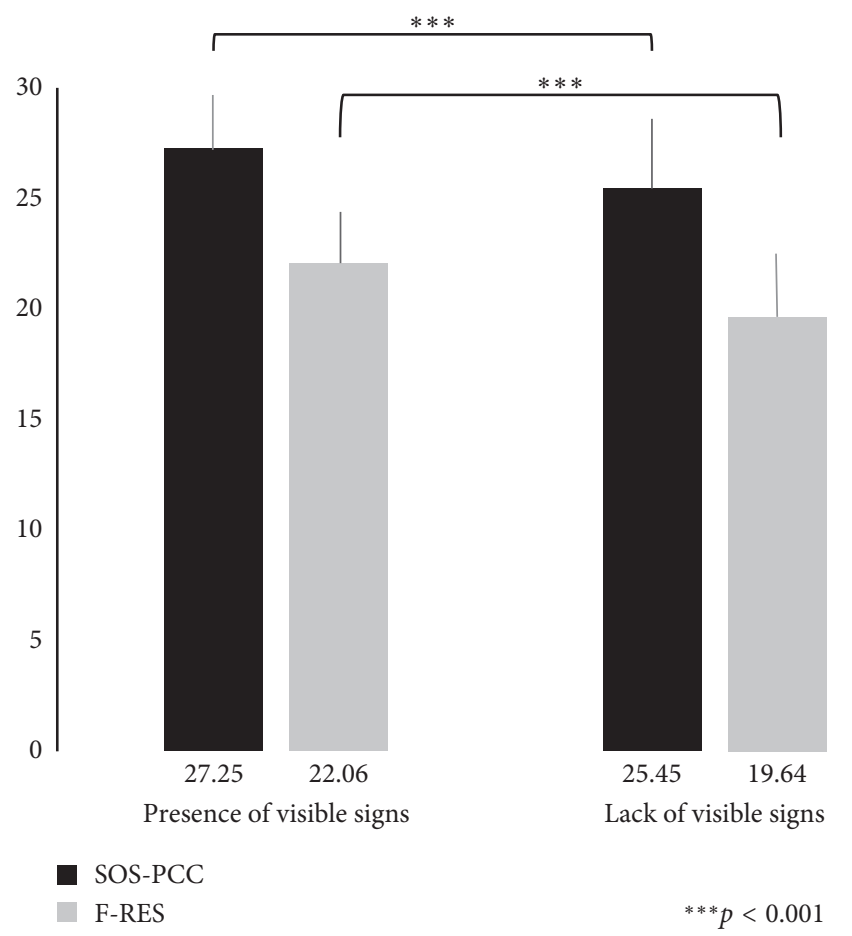

Figure 2: Influence of pain visibility on patient-centered care and empathy. Patient-centered care and empathy behaviours were observed by external raters. SOS-PCC indicates Sherbrooke Orientation Scale of patient-centered care, and F-RES indicates French version of the Reynolds Empathy Scale.

Interestingly, we found that the clinical experience can influence the behaviours of nurses and physicians. Indeed, caregivers with more years of experience are less likely to change their behaviour were according to the presence or absence of physical visible signs. Many studies have investigated the influence of clinical experience on PCC and 
TABLE 2: Influence of clinical experience on caregivers' behaviours in the presence or absence of physical visible signs.

\begin{tabular}{lccc}
\hline & $\begin{array}{c}\text { Similar } \\
\text { ratings }\end{array}$ & $\begin{array}{c}\text { Different } \\
\text { ratings }\end{array}$ & $\begin{array}{c}p \\
\text { value }\end{array}$ \\
\hline $\begin{array}{l}\text { Patient-centered } \\
\text { behaviour } \\
\quad n\end{array}$ & 14 & & \\
$\quad \begin{array}{l}\text { Clinical experience, } \\
\text { years (SD) }\end{array}$ & $24.64(8.68)$ & $17.29(10.36)$ & 0.03 \\
$\begin{array}{l}\text { Empathetic behaviour } \\
n\end{array}$ & 20 & 28 & \\
$\quad \begin{array}{l}\text { Clinical experience, } \\
\text { years (SD) }\end{array}$ & $20.35(9.73)$ & $19.18(11.05)$ & 0.23 \\
\hline
\end{tabular}

$\mathrm{SD}=$ standard deviation.

empathy [44, 45], but little is known about how clinical experience could modulate these variables. A brain imaging study showed that clinical exposure could reduce empathy for pain [46]. More specifically, the authors investigated cortical activity among physicians and control subjects exposed to a series of visual stimuli with body parts in painful and painless situations. Their results indicated that the cortical structures associated with empathy for pain were significantly activated in the control subjects, but not in physicians. It is suggested that these observations are the consequence of a protective regulatory mechanism in people who work daily with pain in order to prevent their distress [46]. Thus, it is possible to believe that the behaviours of the most experienced caregivers are less influenced by the presence of visible signs of pain. However, our results show no difference in PCC and empathy levels according to the clinical experience $(p>0.05)$. It is also possible that the most experienced caregivers are more aware of the impact of pain visibility, and they consider this potential bias in their interventions.

One potential limitation of our study is the lack of a real interaction between participants and patients in the videos. As a result of its methodological advantages and low cost, we used standardized videos of real chronic pain patients. However, the observation scales used for the assessment of PCC and empathetic behaviours were adapted for this kind of situation. Another potential limitation is the reactivity bias. It is possible that participants have positively modified their answers regarding the treatment plan that they would provide in reality. However, many efforts have been made to mitigate this potential bias. First, the participants did not know the variables under study at the time of data collection. Secondly, the questionnaires were distributed at the end of the experimental session in order to avoid a modification in behaviour, conscious or not, from participants.

\section{Conclusions}

In the last years, several studies have shown that practice orientation and empathy of professional caregivers could be influenced by many patients' factors $[43,45,46]$, and our results are consistent with these previous findings. To our knowledge, this is the first study showing a direct influence of visible physical signs on PCC and empathy in the context of chronic pain management. Those observations are to be considered, since chronic pain patients often do not show any apparent physical signs. Thus, these patients are more likely to receive suboptimal pain management, more disease centered and devoid of empathic behaviour. This raises the importance of educating caregivers and future caregivers to these unconscious biases and the potential impact on chronic pain patients. The impact of the presence or absence of apparent physical signs must also be considered for research in chronic pain since it is a potential confounding factor. Future studies could utilize real clinical encounters and other populations to replicate these results.

\section{Data Availability}

The data used to support the findings of this study are available from the corresponding author upon request.

\section{Disclosure}

This work is part of a doctoral study. This article is based on the thesis "Paul-Savoie E, Identification des caractéristiques des soignants liées à l'utilisation d'une approche centrée sur le patient dans un contexte de douleur chronique" (published doctoral thesis; https://doi.org/10.13039/100009874) (Université de Sherbrooke, Sherbrooke, Canada, https://savoirs. usherbrooke.ca/handle/11143/6974).

\section{Conflicts of Interest}

The authors declare that there are no conflicts of interest.

\section{Acknowledgments}

E. Paul-Savoie had at the time of the study a doctoral scholarship from the Canadian Institute of Health Research (CIHR) and was supported by the medicine and health science faculty of the Universite de Sherbrooke. P. Bourgault was at the time of the study a Junior 1 Clinician Investigator with the Quebec Fund for Health Research (FRQS) and a researcher at the Centre de recherche du Centre hospitalier universitaire de Sherbrooke (CR-CHUS). S. Potvin was at the time of the study a Junior 1 Young Investigator from the FRQS and was supported by the Centre de recherche de l'Institut universitaire en santé mentale de Montréal and the Louis-H Lafontaine Hospital Foundation. E. Gosselin had doctoral scholarships from the FRQS, the Ministère de l'éducation supérieure, de la recherche et des sciences (MESRS)-Universités, and the Réseau de recherche en interventions en sciences infirmières du Québec. S. Lafrenaye was supported by the medicine and health science faculty of the Universite de Sherbrooke and a researcher at the CRCHUS.

\section{References}

[1] D. S. Goldberg and S. J. McGee, "Pain as a global public health priority," BMC Public Health, vol. 11, no. 1, p. 770, 2011. 
[2] R. A. Elzahaf, O. A Tashani, B. A Unsworth et al., "The prevalence of chronic an analysis of countries with a human development index less than 0.9: a systematic review without meta-analysis," Current Medical Research and Opinion, vol. 28, no. 7, pp. 1221-1229, 2012.

[3] D. J. Gaskin and P. Richard, "The economic costs of pain in the United States," Journal of Pain, vol. 13, no. 8, pp. 715-724, 2012.

[4] P. A. Pizzo and N. M. Clark, "Alleviating suffering 101-pain relief in the United States," New England Journal of Medicine, vol. 366, no. 3, pp. 197-199, 2012.

[5] L. Jacobson and A. J. Mariano, "General considerations of chronic pain," in Bonica's Management of Pain, J. D. Loeser, S. H. Butler, R. Chapman, and D. C. Turk, Eds., pp. 241-254, Lippincott Williams \& Wilkins, Philadelphia, PA, USA, 2001.

[6] J. Carter, A. C. Watson, and P. V. Sminkey, "Pain management: screening and assessment of pain as part of a comprehensive case management process," Professional Case Management, vol. 19, no. 3, pp. 126-134, 2014.

[7] C. M. Goertz, S. A. Salsbury, C. R. Long et al., "Patientcentered professional practice models for managing low back pain in older adults: a pilot randomized controlled trial," $B M C$ Geriatrics, vol. 17, pp. 235-248, 2017.

[8] K. Bilodeau, S. Dubois, and J. Pepin, "Contribution des sciences infirmières au développement des savoirs interprofessionnels," Recherches en soins infirmiers, vol. 113, no. 2, pp. 43-50, 2013.

[9] C. Hudon, M. Fortin, J. L. Haggerty et al., "Measuring patients' perceptions of patient-centered care: a systematic review of tools for family medicine," Annals of Family Medicine, vol. 9, no. 2, pp. 155-164, 2011.

[10] B. McCormack and T. McCance, "Developing a conceptual framework for person-centred nursing," Journal of Advanced Nursing, vol. 56, no. 5, pp. 472-479, 2006.

[11] M. M. Alamo, R. R. Moral, and L. A. P. de Torres, "Evaluation of a patient-centred approach in generalized musculoskeletal chronic pain/fibromyalgia patients in primary care," Patient Education and Counseling, vol. 48, no. 1, pp. 23-31, 2002.

[12] D. B. Monsivais and J. C. Engebretson, "Cultural cues: review of qualitative evidence of patient-centered care in patients with nonmalignant chronic pain," Rehabilitation Nursing, vol. 36, no. 4, pp. 166-171, 2011.

[13] T. Taverner, S. J. Closs, and M. Briggs, "The journey to chronic pain : a grounded theory of older adults' experience of pain associated with leg ulceration," Pain Management Nursing, vol. 15, pp. 186-198, 2014.

[14] M. S. Krasner, R. M. Epstein, H. Beckman et al., “Association of an educational program in mindful communication with burnout, empathy, and attitudes among primary care physicians," JAMA, vol. 302, no. 12, pp. 1284-1293, 2009.

[15] S. A. Passalacqua and C. Segrin, "The effect of resident physician stress, burnout, and empathy on patient-centered communication during the long-call shift," Health Communication, vol. 27, no. 5, pp. 449-456, 2011.

[16] M. Hojat, J. S. Gonnella, T. J. Nasca et al., "Physician empathy: definition, components, measurement, and relationship to gender and specialty," American Journal of Psychiatry, vol. 159, no. 9, pp. 1563-1569, 2002.

[17] E. B. Larson and X. Yao, "Clinical empathy as emotional labor in the patient-physician relationship," JAMA, vol. 293, no. 9, pp. 1100-1106, 2005.

[18] M. Neumann, M. Wirtz, E. Bollschweiler et al., "Determinants and patient-reported long-term outcomes of physician empathy in oncology: a structural equation modelling approach,"
Patient Education and Counseling, vol. 69, no. 1-3, pp. 63-75, 2007.

[19] D. P. Rakel, T. J. Hoeft, B. P. Barrett et al., "Practitioner empathy and the duration of the common cold," Family Medicine, vol. 41, no. 7, pp. 494-501, 2009.

[20] L. Canovas, A. J. Carrascosa, M. Garcia et al., "Impact of empathy in the patient-doctor relationship on chronic pain relief and quality of life: a prospective study in Spanish pain clinics," Pain Medicine, vol. 19, no. 7, pp. 1304-1314, 2017.

[21] P. Haidet, J. E. Dains, D. A. Paterniti et al., "Medical student attitudes toward the doctor-patient relationship," Medical Education, vol. 36, no. 6, pp. 568-574, 2002.

[22] S. Parsons, G. Harding, A. Breen et al., "Will shared decision making between patient with chronic musculoskeletal pain and physiotherapists, osteopaths and chiropractors improve patient care?," Family Practice, vol. 29, no. 2, pp. 203-212, 2012.

[23] R. M. Epstein, P. Franks, K. Fiscella et al., "Measuring patientcentered communication in patient-physician consultations: theoretical and practical issues," Social Science and Medicine, vol. 61, no. 7, pp. 1516-1528, 2005.

[24] K. D. Bertakis and R. Azari, "Determinants and outcomes of patient-centered care," Patient Education and Counseling, vol. 85, no. 1, pp. 46-52, 2011.

[25] S. Willems, S. De Maesschalck, M. Deveugele et al., "Socioeconomic status of the patient and doctor-patient communication: does it make a difference?," Patient Education and Counseling, vol. 56, no. 2, pp. 139-146, 2005.

[26] L. C. Zandbelt, E. M. Smets, F. J. Oorf et al., "Determinants of physicians' patient-centred behaviour in the medical specialist encounter," Social Science and Medicine, vol. 63, no. 4, pp. 899-910, 2006.

[27] J. Decety, S. Echols, and J. Correll, "The blame game: the effect of responsibility and social stigma on empathy for pain," Journal of Cognitive Neuroscience, vol. 22, no. 5, pp. 985-997, 2009.

[28] D. B. Monsivais, "Decreasing the stigma burden of chronic pain," Journal of the American Association of Nurse Practitioners, vol. 25, no. 10, pp. 551-556, 2013.

[29] J. M. Hemmerdinger, S. Stoddart, and A. Lilford, "A systematic review of tests of empathy in medicine," BMC Medical Education, vol. 7, no. 1, p. 24, 2007.

[30] J. Childs, "Clinical resource centers in nursing programs," Nurse Educator, vol. 27, no. 5, pp. 232-235, 2002.

[31] E. Paul-Savoie, P. Bourgault, E. Gosselin et al., "Assessing patient-centered care for chronic pain: validation of a new research paradigm," Pain Research and Management, vol. 20, no. 4, pp. 183-188, 2015.

[32] E. Paul-Savoie, "Identification des caractéristiques des soignants liées à l'utilisation d'une approche centrée sur le patient dans un contexte de douleur chronique,"Sherbrooke, Canada, (Published doctoral thesis).

[33] E. Paul-Savoie, P. Bourgault, E. Gosselin et al., "Assessing patient-centered care: validation of the French Version of the Patient-Practitioner Orientation Scale (PPOS)," European Journal for Person Centered Healthcare, vol. 3, no. 3, pp. 295-302, 2015.

[34] E. Krupat, S. L. Rosenkranz, C. M. Yeager et al., "The practice orientations of physicians and patients: the effect of doctorpatient congruence on satisfaction," Patient Education and Counseling, vol. 39, no. 1, pp. 49-59, 2000.

[35] C. A. McHorney, J. E. Ware, J. F. R. Lu et al., "The MOS 36item short-form health survey (SF-36): III. Tests of data 
quality, scaling assumptions, and reliability across diverse patient groups," Medical Care, vol. 32, no. 1, pp. 40-66, 1994.

[36] M. Hojat, J. S. Gonnella, S. Mangione et al., "Physician empathy in medical education and practice: experience with the Jefferson scale of physician empathy," Seminars in Integrative Medicine, vol. 1, no. 1, pp. 25-41, 2003.

[37] E. Gosselin, E. Paul-Savoie, S. Lavoie et al., "Reliability and validity of the French version of the Reynolds Empathy Scale in nursing," Journal of Nursing Measurement, vol. 23, pp. E16-E26, 2015.

[38] C. M. H. Chan and W. A. W. Ahmad, "Differences in physician attitudes towards patient-centredness: across four medical specialties," International Journal of Clinical Practice, vol. 66, no. 1, pp. 16-20, 2011.

[39] A. M. Grilo, M. C. Santos, J. S. Rita et al., "Assessment of nursing students and nurses' orientation toward patientcenteredness," Nurse Education Today, vol. 34, no. 1, pp. 35-39, 2014.

[40] S. K. Fields, M. Hojat, J. S. Gonnella et al., "Comparisons of nurses and physicians on an operational measure of empathy," Evaluation and the Health Professions, vol. 27, no. 1, pp. 80-94, 2004.

[41] P. K. Graugaard, K. Holgersen, H. Eide et al., "Changes in physician-patient communication from initial to return visits: a prospective study in a haematology outpatient clinic," $\mathrm{Pa}$ tient Education and Counseling, vol. 57, no. 1, pp. 22-29, 2005.

[42] A. Allaz, Le Messager Boiteux: Approche Pratique des Douleurs Chroniques Rebelles, Genève: Médecine et Hygiène, in French, Lavoisier S.A.S, Chêne-Bourg, Switzerland, 2003.

[43] P. Riva, P. Rusconi, L. Montali et al., "The influence of anchoring on pain judgement," Journal of Pain and Symptom Management, vol. 42, no. 2, pp. 265-277, 2011.

[44] M. Neumann, F. Edelhauser, D. Tauschel et al., "Empathy decline and its reasons: a systematic review of studies with medical students and residents," Academic Medicine, vol. 86, no. 8, pp. 996-1009, 2011.

[45] Z. Tsimtsiou, A. Benos, A. A. Garyfallos et al., "Predictors of physicians' attitudes toward sharing information with patients and addressing psychosocial needs: a cross-sectional study in Greece," Health Communication, vol. 27, no. 3, pp. 257-263, 2012.

[46] Y. Cheng, C. P. Lin, H. L. Li et al., "Expertise modulates the perception of pain in others," Current Biology, vol. 17, no. 19, pp. 1708-1713, 2007. 


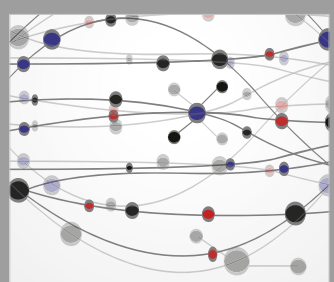

The Scientific World Journal
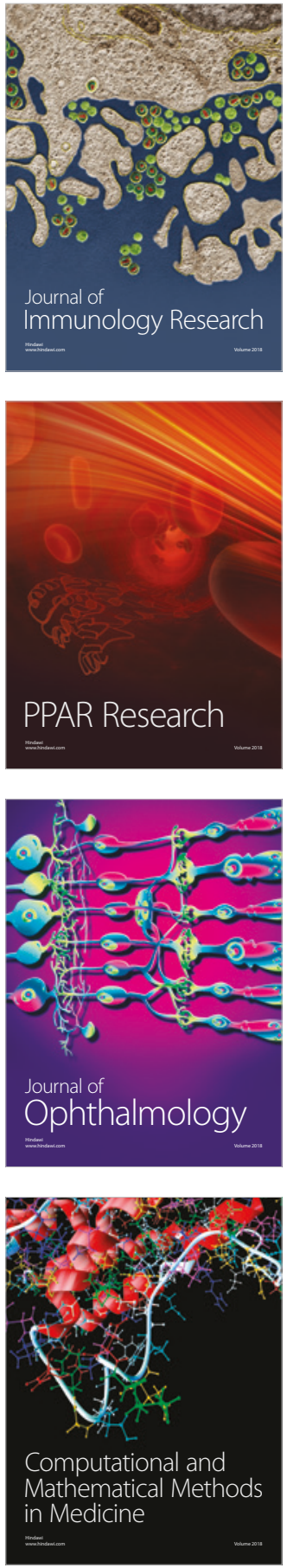

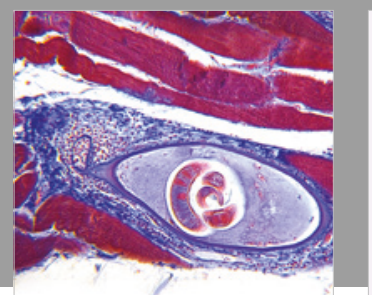

Gastroenterology Research and Practice

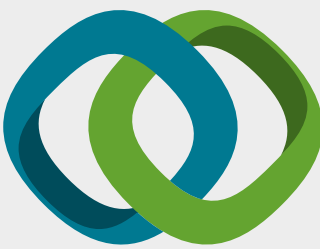

\section{Hindawi}

Submit your manuscripts at

www.hindawi.com
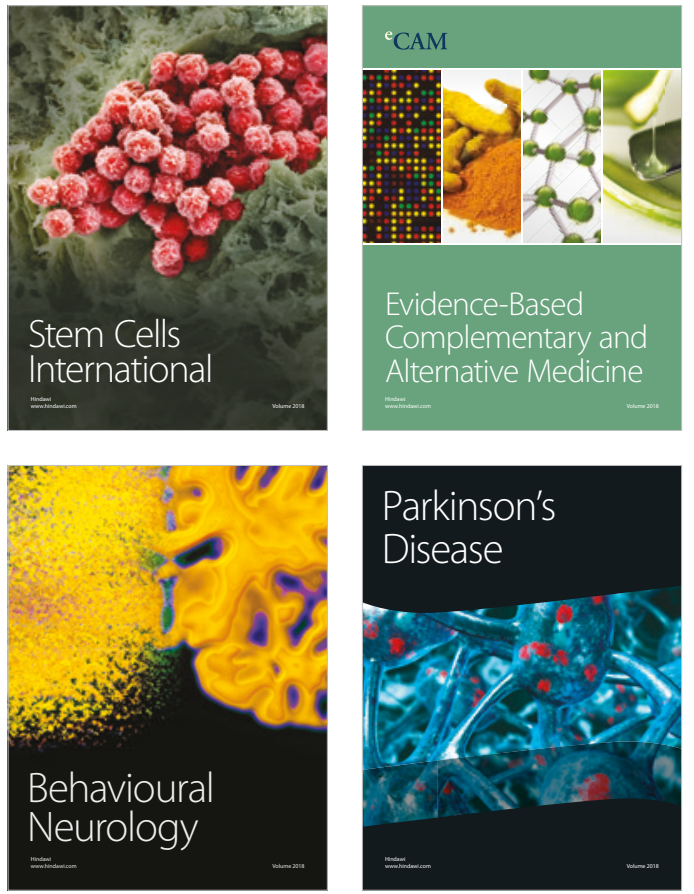

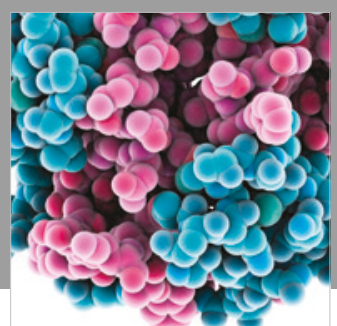

ournal of

Diabetes Research

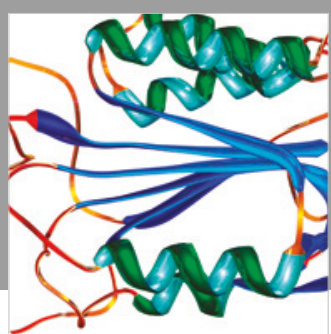

Disease Markers
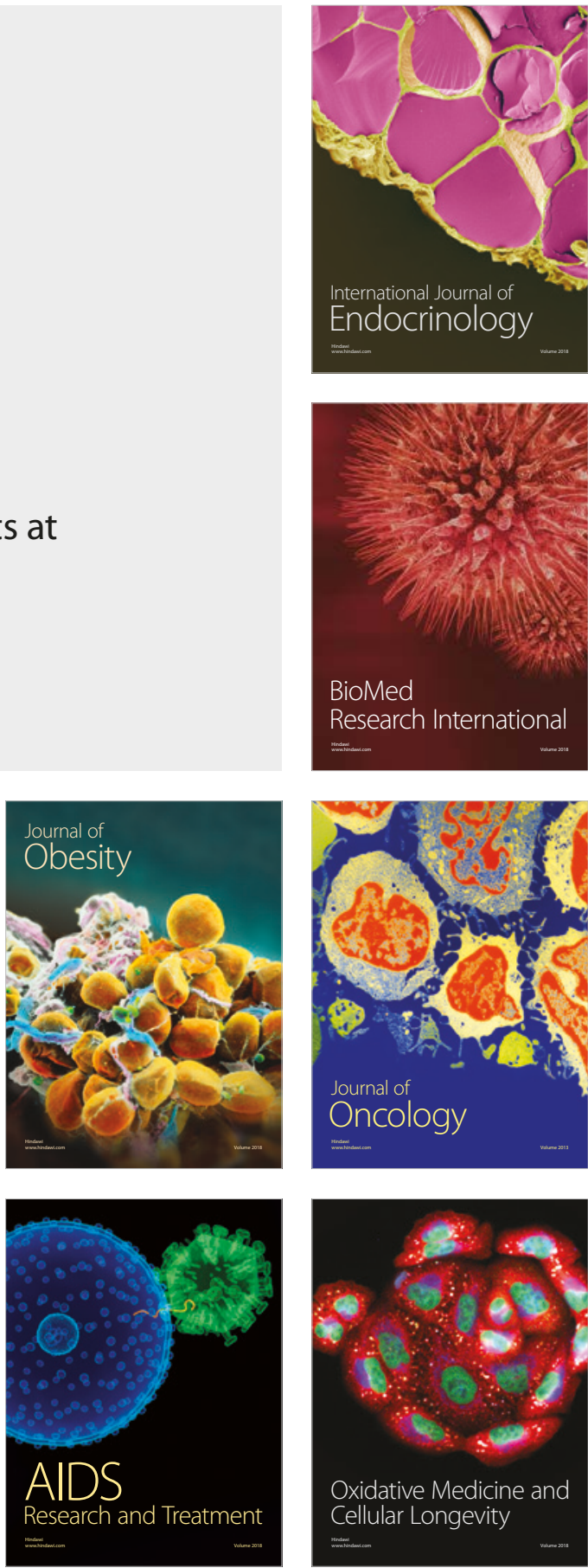